\title{
Risk sharing, sorting, and early contracting
}

Hao Li; Wing Suen

The Journal of Political Economy; Oct 2000; 108, 5; ABI/INFORM Global pg. 1058

\section{Risk Sharing, Sorting, and Early Contracting}

\author{
Hao Li \\ University of Toronto
}

\section{Wing Suen}

University of Hong Kong

\begin{abstract}
In an assignment market with uncertainty regarding productive ability of participants, early contracting can occur as participants balance risk sharing and sorting efficiency. More promising agents may contract early with each other because insurance gains outweigh sorting inefficiency, whereas less promising agents wait. It can also happen in equilibrium that more promising job applicants contract early with less promising firms. Such worker-driven equilibria may arise when applicants are more risk-averse, have greater uncertainty regarding their quality, or face a tighter market and when production exhibits increasing returns to firms' qualities. Early contracting then unambiguously hurts the more promising firms that choose to wait.
\end{abstract}

\section{Introduction}

Entry-level professional labor markets, such as the market for fresh law school graduates and the market for medical interns, are characterized by two distinguishing features. First, match considerations are paramount. Productivity of a law school graduate depends crucially on which law firm he or she is to work with. Second, employment appointments

We are grateful for the comments received at the 1997 Far Eastern Econometrics Society meetings in Hong Kong and at a seminar at the Chinese University of Hong Kong, and especially those of the editor and the anonymous referees. Joseph Lai provided us useful information about college admission programs based on his personal experience. This work is supported by a grant from the Research Grants Council of the Hong Kong Special Administrative Region, China (project HKU7155/98H), 
can be made prior to attainment of professional qualifications by applicants. Coordination of the timing of appointments has been the core issue of attempts to develop market institutions. It is well known that law firms use summer associate programs to attract second-year or even first-year law school students in anticipation of tough competition for highly qualified graduates (see Roth and Xing [1994] and the references therein). Since summer associate programs last for a substantial period and positions are often limited, they amount to early appointments by the law firms. Successful summer associate programs are often run by prestigious law firms. Moreover, there is indication that they mainly attract promising students, for the majority of the students who have gone through the programs receive offers to stay with the firms.

The pattern of early contracting is perhaps clearer in the market for college freshmen, where the recent growth of early college admission programs has attracted the attention of students and educators. Regular admission programs start in December, when applicants are in their senior year of high school, and end in May the following year. Early admission programs start in the junior year, and admission decisions are made before December. According to a report that appeared in the New York Times (February 14, 1996), top colleges have begun to fill more slots with students who apply early. The number of colleges that have adopted early admission programs stood at 471 in 1996, most of them top colleges in the country. Moreover, students who apply early tend to be the good ones. This can be seen from a comparison of the admission/ application ratio in early and regular admission programs. For example, at the University of Chicago, the admission/application ratio was 60 percent for the class of 1995 in the early program, compared to only about 6 percent in regular programs of top colleges for the same year. The report also noted that some schools (e.g., Stanford University) have "binding" early admission programs, where successful applicants are asked not to apply to other colleges later through the regular programs, whereas other programs (called "early action" as opposed to early admission) such as the one at the University of Chicago do not have such a restriction.

A common feature of these examples of early contracting is that participants are acutely aware of the cost of signing contracts before adequate information can be obtained about the qualifications of applicants as well as the desirability of potential employers. This is especially the case when the date of early appointments advances to well before the date of attainment of professional qualifications by job applicants, such as in the market for law school graduates, where promising students become summer interns at prestigious law firms after their first year in law school, and in the market for medical school graduates before the enforcement of the uniform appointment date (Roth 1984). But 
even in the case of college admissions, early admissions create a significant amount of uncertainty on both sides of the market compared to regular admissions. The article in the New York Times reported that many educators worry that "the early admission process may push 17-year-olds to make an important decision too soon," before they have a clear idea which universities will better suit their educational needs. On the other hand, large-scale early admission programs require systematic information about the quality of students, but part of the information is readily available only a year later at the beginning of the regular admission season. A promising high school junior may turn out to have a bad senior year that lowers the overall grade point average or a successful one that makes the student a favorite of all top colleges. For all the uncertainty, however, early admission programs allow some students to lock in a college of choice and therefore to "relieve the pressure of insanity" of college admission, and allow some colleges to lock in desirable students and avoid those "who were just collecting trophies."

This paper tries to address some of the important questions about early appointments. Do early appointments signal a trend toward a longterm stable situation, or are they just a passing phenomenon? Why is it that top law firms and top colleges have more interest in adopting early appointments and top applicants are more eager to apply early? How do nonbinding early admission programs change the incentives for early admissions? What determines the timing of early appointments? Can early contracting take a form different from the "top-clearing" pattern? How do early appointments affect the welfare of different groups of the market participants?

We present an equilibrium model of competitive early contracting, built on the familiar assignment model of Koopmans and Beckmann (1957) and Shapley and Shubik (1972). The crucial departure is that at an early appointment date market participants do not have complete information regarding their productive ability. This uncertainty may motivate some agents to contract with each other before uncertainty is resolved in order to reduce the risks. But since the information necessary to achieve efficient assignments is incomplete at the time of contracting, matches formed ex ante are likely to be inefficient ex post. There is a trade-off between risk sharing and efficient assignments.

In Section II, we first illustrate the trade-off in early contracting between risk sharing and efficient sorting, with plausibly specified terms of early contracts and pattern of early matching. We show that in a competitive equilibrium framework, risk aversion of some market participants is a necessary condition for early contracting. A formal definition of early contracting as a competitive equilibrium is then presented, where the terms of contracts and the pattern of matching are part of the equilibrium. 
Section III provides sufficient conditions that early contracting is "positive assortative" in that, among agents signing early contracts, matches are formed between workers and firms that are ranked equally according to their prospects. These conditions are met if firms are risk-neutral because in this case the complementarity between firms and workers dominates competition for partners in the ex ante market. Then we analyze a particular positive assortative pattern of early contracting called "top clearing," where more promising agents sign early contracts and less promising agents wait until the uncertainty regarding ability is resolved. A fully explicit model is provided to illustrate how top-clearing early contracting occurs because more promising workers face greater payoff risks by waiting for the ex post market whereas workers and firms of all prospects gain equally from efficient ex post sorting. The benefits from risk sharing dominate the gains from efficient sorting for more promising workers and firms, and the opposite occurs for less promising agents. Comparative statics analysis of the model shows that the extent of early contracting is positively related to the degree of risk aversion of workers and to the degree of uncertainty regarding ability. We show that if early contracts are nonbinding in the sense that agents can renegotiate the early contracts they signed by reentering the market after uncertainty is resolved, then early contracting will be pervasive. These results explain that the extent of early college admissions is limited by the amount of new information that colleges and students can learn about each other in the senior year of high school, but nonbinding agreements in these programs can increase their popularity. Finally, the model is extended to a more generally dynamic framework in which uncertainty regarding ability is resolved gradually. We show that an increase in risk aversion will push the equilibrium contracting dates earlier.

Section IV examines the equilibrium possibility of a non-top-clearing pattern of early matching, namely, the "worker-driven" pattern, where promising workers are driven to contract early with firms that are not promising. This pattern occurs in equilibrium when the trade-off between risk sharing and efficient sorting varies with the prospects of workers and firms in an asymmetric way: for more promising workers the gains from risk sharing dominate, but for more promising firms the gains from efficient sorting dominate. We show that worker-driven contracting is more likely to occur in a competitive equilibrium if applicants are more risk-averse than firms, have greater uncertainty regarding their quality, or face a tighter market and if the production function exhibits increasing returns to firms' productive characteristics. A full model is developed to illustrate why in equilibrium promising firms are willing to let other firms outbid them for promising workers with early contracts: risk-averse workers are eager to obtain insurance from ex ante contracts and higher types of workers have more at risk from waiting than lower 
types of workers, whereas risk-neutral firms are not so eager to sign ex ante contracts and higher types of firms have stronger incentives to wait and exploit the efficiency gains made possible by a production function with increasing returns to firms' productive characteristics. We show that early contracting in this case unambiguously hurts the more promising firms that choose to wait, explaining why early contracting in entrylevel professional labor markets often provokes firms' efforts to prohibit it (Roth and Xing 1994). Section V of the paper discusses the related literature.

\section{Early Contracting as a Competitive Equilibrium}

Our model of early contracting has two possible appointment dates, ex ante and ex post. Production begins after the second date. Output of a pair of a worker and a firm is given by the production function $F(X, Y)$, with $F_{1}>0, F_{2}>0$, and $F_{12}>0$, where $X$ and $Y$ denote the ability of the worker and of the firm, respectively. The assumption of positive cross-derivatives $F_{12}$ implies complementarity between the worker and the firm, and is standard in the assignment market literature (see, e.g., Becker 1981). Ability is not completely known ex ante; instead each agent knows only his type, which is a probability distribution of ex post ability. We assume that type is public information. Throughout the paper, we assume that distributions of the two random variables $X$ and $Y$ conditional on their respective type are independent.

\section{A. The Trade-off between Risk Sharing and Efficient Sorting}

When agents are risk-averse, the desire to reduce payoff risks in ex post assignments can motivate some of them to contract early, even though this implies a loss of sorting efficiency. The trade-off in early contracting between risk sharing and efficient assignments can be readily illustrated. Suppose that for each type $x$ of workers, the ability $X$ conditional on type $x$ is a random variable with mean $x$ and constant variance $\sigma^{2}$. Similarly, for firms, conditional on type $y$, the ability $Y$ is a random variable with mean $y$ and the same variance $\sigma^{2}$. As in Becker (1981), since $F_{12}>0$, there will be positive assortative matching in the ex post market because the production function exhibits complementarity between the inputs. When the realized distributions of $X$ and $Y$ are identical, if assignments take place after ability is fully revealed, workers of ability $X$ will be matched with firms with the same realized ability $Y=X$. When there is equal sharing of output between the two inputs, a type $x$ worker's expected payoff is one-half of 


$$
E[F(X, X) \mid x] \approx F(x, x)+\frac{F_{11} \sigma^{2}+2 F_{12} \sigma^{2}+F_{22} \sigma^{2}}{2} .
$$

On the other hand, if assignments take place before ability is revealed, ex post ability of the two inputs in any pair will be different. Under the assumption that type $x$ workers are matched to type $y=x$ firms with equal sharing of the random output, a type $x$ worker's expected payoff is one-half of

$$
E[F(X, Y) \mid x, x] \approx F(x, x)+\frac{F_{11} \sigma^{2}+F_{22} \sigma^{2}}{2} .
$$

Since $F_{12}>0$, expected payoff is lower if agents contract early.

While waiting until ability is revealed induces higher output and payoff through more efficient assignments, it also exposes individuals to larger risks. With positive assortative matching in the ex post market, a higher realized level of an agent's ability raises his payoff both directly through the agent's own greater contribution to output and indirectly through a greater contribution from a partner of a higher realized ability. Similarly, a low-ability agent will produce low output because of low productivity and because of assignment to a low-ability partner. Taking a first-order Taylor approximation to $F(X, X)$ around $x$, we find that the variance of a type $x$ worker's payoff is one-quarter of

$$
\operatorname{Var}[F(X, X) \mid x] \approx F_{1}^{2} \sigma^{2}+2 F_{1} F_{2} \sigma^{2}+F_{2}^{2} \sigma^{2} .
$$

The middle term in this equation arises from the positive correlation between $X$ and $Y$ under positive assortative matching in the ex post market. If people are matched ex ante by their types rather than by their realized ability, such positive correlation will be removed because $X$ and $Y$ are conditionally independent. The variance of a type $x$ worker's payoff becomes one-quarter of

$$
\operatorname{Var}[F(X, Y) \mid x, x] \approx F_{1}^{2} \sigma^{2}+F_{2}^{2} \sigma^{2} .
$$

The four equations above illustrate the trade-off between risk sharing and efficient assignments. If agents are sufficiently risk-averse, they may be willing to sacrifice the output gains from efficient matching and sign contracts before ability is fully known. The trade-off between risk sharing and efficient sorting is related to the literature on assignment models of earnings distribution (e.g., Rosen 1981, 1982; Kremer 1993; Sattinger 1993). When the production function exhibits complementarity in individual ability, assignment by ability results in a distribution of earning that is more dispersed than the distribution of ability. While the result has long been recognized to be a factor in generating income inequality (e.g., Tinbergen 1951), its implications for individuals' demand for insurance have been ignored. Early contracting analyzed in our paper is 
an insurance device by which individuals reduce the risks inherent in assignment markets.

If all agents are risk-neutral, there is no trade-off between risk sharing and efficient sorting, and waiting for the ex post market is always more advantageous to contracting early for all types. One may wonder whether this conclusion depends on our assumption of equal sharing both in ex ante contracts and in the ex post market, or on the restriction to contracting between agents of equal types. It turns out that this is not the case, as long as the terms and the pattern of contracting are competitively determined in both the ex ante and the ex post markets. To make the formal argument, suppose that the equilibrium ex post wage profile for workers is $C(X)$ and the ex post profit function for firms is $D(Y)$. Since the ex post assignment market is competitive, if a worker with realized ability $X$ is not an equilibrium partner of a firm with realized ability $Y$, then they cannot gain by matching with each other. This implies that

$$
C(X)+D(Y) \geq F(X, Y),
$$

where equality holds if and only if the worker and the firm are equilibrium partners. Taking expectations on both sides with respect to $X$ conditional on any type $x$ and then with respect to $Y$ conditional on any type $y$, we have

$$
E[C(X) \mid x]+E[D(Y) \mid y]>E[F(X, Y) \mid x, y],
$$

where strict inequality results because with positive probability a type $x$ worker and a type $y$ firm will not turn out to be equilibrium partners in the ex post market. In a competitive ex ante market, a type $y$ firm must pay at least $E[C(X) \mid x]$ to induce risk-neutral type $x$ workers to sign early contracts, but then its expected profits are at most $E[F(X, Y) \mid x, y]-E[C(X) \mid x]$, which by the condition above is lower than the profits $E[D(Y) \mid y]$ that it can get by waiting for the ex post market. Thus early contracting cannot occur for any pair of risk-neutral worker and firm.

\section{B. Early Contracting Equilibrium}

In this paper, we apply competitive analysis to the ex ante market in which agents choose between contracting early and waiting for the ex post market. Patterns of early contracting (who signs up with whom) and the terms of early contracts are determined in a competitive equilibrium. To formally define early contracting as a competitive equilibrium, let $T_{1}$ and $T_{2}$ be the set of all types of workers and firms, respectively, and let $S_{1}$ be a subset of $T_{1}$ and $S_{2}$ be a subset of $T_{2}$ with the same measure, representing the sets of agents that contract early. The ex ante 
distributions and the conditional distributions of types $x \in T_{1} \backslash S_{1}$ and types $y \in T_{2} \backslash S_{2}$ determine the distributions of ability of the two inputs in the ex post market. Given a competitive equilibrium in this ex post market, for each $x \in T_{1}$, let $U_{*}(x)$ be the expected utility of type $x$ from waiting, determined by type $x$ 's conditional distribution of ability and the payoff functions in the competitive equilibrium. Similarly, for each $y \in T_{2}$, define $V_{*}(y)$ as the expected utility of type $y$ from waiting.

Definition 1. An early contracting equilibrium is the sets of types $S_{1}$ and $S_{2}$, a one-to-one assignment function $a(y)$ that gives type $x \in S_{1}$ for each $y \in S_{2}$, and utilities $u_{*}(x)$ and $v_{*}(y)$ for all $x \in T_{1}$ and $y \in T_{2}$ such that (i) $u_{*}(x) \geq U_{*}(x)$ for each $x \in S_{1}$ and $v_{*}(y) \geq V_{*}(y)$ for each $y \in$ $S_{2}$; (ii) $u_{*}(x)=U_{*}(x)$ for each $x \in T_{1} \backslash S_{1}$ and $v_{*}(y)=V_{*}(y)$ for each $y \in T_{2} \backslash S_{2}$; (iii) for each $y \in S_{2}$, there exists an income-sharing contract between a type $a(y) \in S_{1}$ worker and a type $y$ firm that yields expected utility $u_{*}(a(y))$ to type $a(y)$ and $v_{*}(y)$ to type $y$; and (iv) for each $y \in$ $T_{2}$, there is no type $x \in T_{1}$ such that a contract between type $x$ and type $y$ yields expected utility $u_{*}(x)$ to type $x$ and an expected utility strictly greater than $v_{*}(y)$ to type $y$.

The early contracting equilibrium defined above can be compared to the core in assignment markets with no uncertainty. The core is defined as an assignment function and payoffs to the participants such that no group of participants can Pareto-improve the payoffs of its members by forming pairwise matches among themselves. In an early contracting equilibrium defined above, no pair of agents can form an early match that Pareto-improves on their equilibrium utilities. There are two important differences between the core and the early contracting equilibrium. In the core of assignment markets with no uncertainty, it is customarily assumed that the option of not being assigned yields a fixed payoff to any agent. In the early contracting equilibrium of the ex ante market, the option that every agent has is remaining in the market, which yields different expected utilities to agents with different conditional distributions of ability. Moreover, these expected utilities are part of the early contracting equilibrium to be determined endogenously. The other important difference between the core and the early contracting equilibrium is that because of the pairwise nature of the production function, in checking whether the assignment function and associated payoffs form a core, one can consider only pairs of agents for "blocking" coalitions. However, risk sharing is more beneficial if it involves more than two individuals. In the definition of an early contracting equilibrium, we simply do not allow agents to insure each other by forming large coalitions. The early contracting equilibrium can be thought of as the core of the ex ante assignment market, where coalitions larger than pairs are excluded by definition. This restriction on 
possible coalitions is reasonable insofar as only pairwise early contracting is observed in assignment markets.

Just as the core in standard assignment markets can be interpreted as a competitive equilibrium (see Koopmans and Beckmann 1957), the early contracting equilibrium defined above can also be interpreted as a competitive equilibrium in the ex ante market. We can think of the equilibrium utility $u_{*}(x)$ of type $x$ as the "price" of the ex ante contract with type $x$. Each type $y$ firm takes as given the function $u_{*}(x)$ and chooses a type $x$ such that the expected utility from contracting with type $x$ is maximized subject to the condition that type $x$ gets expected utility $u_{*}(x)$. The early contracting equilibrium requires that, for all $y \in S_{2}$, the optimal choice of type equal $a(y)$, with the resulting expected utility from ex ante contracting greater than the waiting utility $V_{*}(y)$, and that, for all $y \in T_{2} \backslash S_{2}$, the expected utility be lower than $V_{*}(y)$.

\section{Top-Clearing Early Contracting}

The simple example in Section II shows that in an assignment market with individual uncertainty regarding agents' ability, early contracting can occur before the uncertainty is resolved as agents trade off insurance gains from ex ante contracting against inefficiency of assignments. However, the contracting-waiting decisions in the ex ante market and the terms of contracts in both the ex ante and ex post markets have to be determined competitively. To do so, we need to impose some structure on distributions of ability conditional on types. Prospects of agents are modeled by distributions of ability so that agents of a more promising type have a distribution of ability stochastically dominating that of a less promising type. Throughout the paper, we assume that the degree and the nature of individual uncertainty do not depend on type. ${ }^{1}$ This can be modeled by an additive specification of type. Suppose that $\gamma_{1}$ and $\gamma_{2}$ are two random variables with independent distributions. We assume that the ability conditional on type $x$ is a random variable $X$ equal to $x+\gamma_{1}$. Similarly, the ability $Y$ conditional on type $y$ is given by $y+\gamma_{2}$. Without loss of generality, $\gamma_{1}$ and $\gamma_{2}$ are assumed to have zero mean so that the ex ante type of an agent is his expected ability. Note that the distribution of ability of a higher type dominates that of a lower type in the sense of first-order stochastic dominance.

\footnotetext{
1 This assumption precludes consideration of markets in which the match quality of participants depends only on relative ranking, such as the market for the arrangement of college football bowls (Roth and Xing 1994). In these markets, higher types are likely to face greater down-side risks and have incentives to enter early contracts, whereas lower types tend to wait and take their chances.
} 


\section{A. Positive Assortative Early Contracting}

Complementarity in the production function implies that in the ex post competitive market equilibrium matching is positive assortative in the sense that lower-ability firms cannot outbid higher-ability firms for higher-ability workers. Is competitive early contracting positive assortative in our model? The answer is yes under quite general assumptions about risk attitudes and the production function.

Throughout the paper, we maintain the assumption that the utility function of workers takes the mean-variance form

$$
U(C)=E[C]-r_{1} \operatorname{Var}(C),
$$

and the utility function of firms is similarly given by

$$
V(C)=E[C]-r_{2} \operatorname{Var}(C) .
$$

The parameters $r_{1}$ and $r_{2}$ are nonnegative numbers that indicate degrees of risk aversion of the two inputs. When there is a linear sharing rule between a type $x$ worker and a type $y$ firm, an optimal risk-sharing contract allocates $\lambda F(X, Y)+c$ to the worker and $(1-\lambda) F(X, Y)-c$ to the firm, where $\lambda=r_{2} /\left(r_{1}+r_{2}\right)$ and $c$ is a transfer payment. Then the expected utility of the type $x$ worker is

$$
u(x, y)=\frac{r_{2}}{r_{1}+r_{2}} E[F(X, Y) \mid x, y]+c-r_{1}\left(\frac{r_{2}}{r_{1}+r_{2}}\right)^{2} \operatorname{Var}[F(X, Y) \mid x, y],
$$

and the expected utility of the type $y$ firm is

$$
v(x, y)=\frac{r_{1}}{r_{1}+r_{2}} E[F(X, Y) \mid x, y]-c-r_{2}\left(\frac{r_{1}}{r_{1}+r_{2}}\right)^{2} \operatorname{Var}[F(X, Y) \mid x, y] .
$$

The sum of the expected utilities is

$$
w(x, y)=E[F(X, Y) \mid x, y)-\left(\frac{r_{1} r_{2}}{r_{1}+r_{2}}\right) \operatorname{Var}[F(X, Y) \mid x, y]
$$

Following Becker (1981), we identify a sufficient condition that rules out "negative" assortative matching in any equilibrium of the ex ante market.

Proposition 1. Suppose that $w(x, y)$ has positive cross-partial derivatives for all $x$ and $y$. Then, in any early contracting equilibrium, there do not exist types $x_{1}<x_{2}$ and types $y_{1}<y_{2}$ such that a type $x_{1}$ worker contracts with a type $y_{2}$ firm and a type $x_{2}$ worker contracts with a type $y_{1}$ firm.

Proof. Suppose that there is such an equilibrium. Let $u_{*}\left(x_{1}\right)$ be the equilibrium utility of type $x_{1}$ and $u_{*}\left(x_{2}\right)$ be the utility of $x_{2}$. Since type 
$y_{2}$ must be better off contracting with type $x_{1}$ than contracting with type $x_{2}$,

$$
w\left(x_{1}, y_{2}\right)-u_{*}\left(x_{1}\right) \geq w\left(x_{2}, y_{2}\right)-u_{*}\left(x_{2}\right)
$$

Similarly,

$$
w\left(x_{2}, y_{1}\right)-u_{*}\left(x_{2}\right) \geq w\left(x_{1}, y_{1}\right)-u_{*}\left(x_{1}\right)
$$

These two inequalities imply

$$
w\left(x_{1}, y_{2}\right)+w\left(x_{2}, y_{1}\right) \geq w\left(x_{1}, y_{1}\right)+w\left(x_{2}, y_{2}\right),
$$

which can be written as

$$
\int_{x_{1}}^{x_{2}} \frac{\partial w\left(x, y_{1}\right)}{\partial x} d x \geq \int_{x_{1}}^{x_{2}} \frac{\partial w\left(x, y_{2}\right)}{\partial x} d x .
$$

This contradicts the assumption that $w(x, y)$ has positive cross-partial derivatives for all $x$ and $y$. Q.E.D.

Under the additive specification of conditional distributions, equation (1) implies that the cross-partial derivative of $w(x, y)$ equals

$$
\begin{gathered}
E\left[F_{12}(X, Y) \mid x, y\right]-\left(\frac{2 r_{1} r_{2}}{r_{1}+r_{2}}\right) \\
\times\left[\operatorname{Cov}\left(F, F_{12} \mid x, y\right)+\operatorname{Cov}\left(F_{1}, F_{2} \mid x, y\right)\right] .
\end{gathered}
$$

It immediately follows from expression (2) that since $F_{12}>0$, the condition in the proposition is satisfied if firms are risk-neutral. ${ }^{2}$ The intuition is simple. When firms are risk-neutral, workers are fully insured in early contracts, and firms contracting early care only about the expected ability of their partners. Since higher types of workers are more likely to have greater ability, complementarity in the production function implies that lower types of firms cannot outbid higher types of firms for higher types of workers. There will be positive assortative matching in the ex ante market as well as in the ex post market.

If firms have a risk preference similar to that of workers, the condition in proposition 1 can still be satisfied by a wide range of production functions. To see this, suppose that $F(X, Y)$ is multiplicatively separable:

$$
F(X, Y)=\theta(X) \phi(Y) \text {. }
$$

Then the two covariance terms in expression (2) are both equal to

\footnotetext{
${ }^{2}$ This conclusion does not depend on either mean-variance representation of the preference of workers or the additive specification of conditional distributions.
} 


$$
\begin{gathered}
E\left[\theta(X) \theta^{\prime}(X) \mid x\right] E\left[\phi(Y) \phi^{\prime}(Y) \mid y\right] \\
-E[\theta(X) \mid x] E\left[\theta^{\prime}(X) \mid x\right] E[\phi(Y) \mid y] E\left[\phi^{\prime}(Y) \mid y\right] .
\end{gathered}
$$

By assumption, $\theta^{\prime}(X)>0$ and $\phi^{\prime}(Y)>0$. If $\theta^{\prime \prime}(X) \leq 0$ and $\phi^{\prime \prime}(Y) \leq 0$, the two covariance terms are nonpositive. Thus a sufficient condition for $w(x, y)$ to have positive cross-partial derivatives is that the production function is multiplicatively separable and exhibits nonincreasing returns to each input. In this case, the total risks of any two early contracts are minimized by matching the higher-type agents together and the lower types together. Of course, even if negative assortative matching reduces total risks because of increasing returns, positive assortative matching can still be the rule in the ex ante market since it is favored by complementarity in the production function.

In a standard assignment market without uncertainty regarding the ability of agents, positive assortative matching implies that higher-ability agents are matched with each other and oversupplied lower-ability agents are unable to outbid and remain unmatched. In contrast, in the ex ante market with uncertainty regarding ability of agents, even when negative assortative contracting is ruled out by proposition 1 , there are many possible patterns of early contracting. For example, lower types of firms may bid for higher types of workers, while higher types of firms decide to remain unmatched in the ex ante market and wait for the ex post market. The possibility of such early contracting equilibria will be addressed in Section IV. Subsection $B$ discusses a positive assortative early contracting pattern called "top-clearing" contracting, where more promising agents on both sides of the ex ante market sign early contracts and less promising ones wait until their ability is fully revealed. Topclearing contracting resembles the unique equilibrium matching pattern in an assignment market without uncertainty and is therefore the natural candidate for early contracting equilibrium.

\section{B. A Model of Top-Clearing Early Contracting}

Now we develop a fully explicit model in which promising agents sign ex ante contracts to insure against payoff risks, while others prefer to wait until their ability is fully revealed. The extent of early contracting and the terms of contracts are explicitly solved and comparative statics results are provided. This model also allows us to discuss the competitive equilibrium when early contracts are nonbinding in the sense that participants are allowed to reenter the ex post market as long as their original partners are compensated. Finally, the model easily generalizes to a dynamic model of more than two periods and helps us understand how gradual resolution of the uncertainty regarding ability determines the timing of early contracting. 
To derive explicit solutions, we assume that the production function $F(X, Y)=X Y$. The distribution of ability $X$ conditional on type $x$ is assumed to be normal with mean $x$ and variance $\sigma^{2}$. Similarly, the conditional distribution for a type $y$ firm is normal with mean $y$ and variance $\sigma^{2}$. The types for the two inputs are distributed identically and continuously over an interval $[\underline{t}, \bar{t}]$. The value of $\underline{t}$ is assumed to be large enough so that the possibility of negative realized ability can be safely ignored.

Since the ex ante distributions of types and the distributions of ability conditional on equal types are identical for the two inputs, the ex post distributions of ability remain identical for the two inputs if equal types are matched in early contracting. Then complementarity in the production function implies that agents with the same realized ability are assigned to each other. Payoffs that sustain this as a competitive equilibrium, where firms with realized ability $Y$ maximize their payoff by choosing workers with ability $X=Y$ and satisfy an additional condition that workers of ability 0 receive zero payoffs, are $X^{2} / 2$ to workers with ability $X$ and $Y^{2} / 2$ to firms with ability $Y$. Therefore, the expected utility for a waiting worker $x$ is

$$
\begin{aligned}
U_{*}(x) & =E\left[\frac{X^{2}}{2} \mid x\right]-r_{1} \operatorname{Var}\left[\frac{X^{2}}{2} \mid x\right] \\
& =\frac{1}{2}\left(x^{2}+\sigma^{2}\right)-\frac{r_{1}}{4}\left(4 x^{2} \sigma^{2}+2 \sigma^{4}\right) .
\end{aligned}
$$

The waiting utility for the firm, $V_{*}(y)$, takes a similar form:

$$
V_{*}(y)=\frac{1}{2}\left(y^{2}+\sigma^{2}\right)-\frac{r_{2}}{4}\left(4 y^{2} \sigma^{2}+2 \sigma^{4}\right) .
$$

The term $\sigma^{2}$ in the first term of equations (4) and (5) represents the gain from efficient assignments due to positive assortative matching after ability is fully revealed. Thus, in this model, the efficiency gains are independent of the agent's type. Note that because the payoff function for workers in the ex post market is convex, higher types of workers face greater payoff risks (the second term in eq. [4]).

For any type $x$ worker who contracts early, there must exist a payoff function $u_{*}(x)$ such that the utility $v(x, y)$ to a type $y$ firm from contracting early with type $x$ is derived from eq. (1) and given by

$$
v(x, y)=x y-\frac{r_{1} r_{2}}{r_{1}+r_{2}}\left(x^{2} \sigma^{2}+y^{2} \sigma^{2}+\sigma^{4}\right)-u_{*}(x)
$$

For matching equal types in the ex ante market to be an equilibrium, 
we need $\partial v(x, y) / \partial x=0$ at $x=y$. This implies a differential equation in $u_{*}(x)$, whose solution is given by

$$
u_{*}(x)=\left(\frac{1}{2}-\frac{r_{1} r_{2} \sigma^{2}}{r_{1}+r_{2}}\right) x^{2}+u_{0},
$$

where $u_{0}$ is a constant of integration to be determined.

Comparing equations (6) and (5), one can see that the conditional variance of $X Y$ is only half the conditional variance of $X^{2}$ when $x=y$. Moreover, the gains from risk reduction rise in agents' type. Since the loss from inefficient assignments does not depend on type, we expect that the higher types have greater incentives to contract early than to wait, suggesting a top-clearing pattern of early contracting instead of "bottom clearing." We hypothesize that individuals of types $x \geq z$ and $y \geq z$ match ex ante whereas those below the critical type $z$ wait. The worker and the firm of the critical type $z$ are indifferent between contracting ex ante with each other and waiting. Therefore,

$$
U_{*}(z)=u_{*}(z)
$$

and

$$
V_{*}(z)=y_{*}(z),
$$

where the function $u_{*}(x)$ is given by equation $(7)$ and the function

$$
v_{*}(y)=y^{2}-\frac{r_{1} r_{2}}{r_{1}+r_{2}}\left(2 y^{2} \sigma^{2}+\sigma^{4}\right)-u_{*}(y),
$$

obtained from equation (6), gives the expected utility to type $y$ from ex ante contracting with type $x=y$. Using equation (7), we can solve the two indifference conditions of type $z$ for the two unknowns, $u_{0}$ and $z$. The solutions are

$$
u_{0}=\frac{\left(r_{2}^{2}-r_{1}^{2}\right) \sigma^{2}}{2\left(r_{1}^{2}+r_{2}^{2}\right)}-\frac{r_{1} r_{2} \sigma^{4}}{2\left(r_{1}+r_{2}\right)}
$$

and

$$
z=\left(\frac{r_{1}+r_{2}}{r_{1}^{2}+r_{2}^{2}}-\frac{\sigma^{2}}{2}\right)^{1 / 2}
$$

The comparative statics for the critical type $z$ are straightforward. ${ }^{3}$ When $r_{1}$ and $r_{2}$ both increase, more agents want to insure against payoff risks and the critical level $z$ falls. More precisely, $z$ decreases if both $r_{1}$ and $r_{2}$

\footnotetext{
${ }^{3}$ We restrict attention to the case in which agents' utility from waiting is increasing in type. This requires $r_{1}$ and $r_{2}$ to be less than $\left(2 \sigma^{2}\right)^{-1}$. This condition also ensures that the solution value of $z$ in eq. (9) is well defined.
} 
increase, with $r_{1}-r_{2}$ kept unchanged. Furthermore, the greater the difference in the degree of risk aversion, the greater the opportunity for Pareto-improving risk sharing. Thus, when $r_{1} \geq r_{2}$, the critical level $z$ decreases if $r_{1}$ increases and $r_{2}$ remains constant. ${ }^{4}$ Finally, an increase in the degree of uncertainty $\sigma^{2}$ will also lower the critical level of ex ante contracting. Thus, in this model, with symmetric uncertainty on the two sides of the market regarding match quality, the gains from risk sharing in early contracts grow faster than the loss due to inefficient assignments as the degree of uncertainty increases.

Comparing equation (7) to equation (4), we find that $u_{*}^{\prime}(x)>U_{*}^{\prime}(x)$. Since $u_{*}(z)=U_{*}(z)$, this means that $u_{*}(x)>U_{*}(x)$ for $x>z$ and $u_{*}(x)<$ $U_{*}(x)$ for $x<z$. Similarly, we can use equations (8) and (5) to show that $v_{*}^{\prime}(y) \geq V_{*}^{\prime}(y)$. ${ }^{6}$ Thus types $x \geq z$ and $y \geq z$ match ex ante with equal types, while those below the critical type $z$ wait. To complete derivation of this model, we need to verify that no pair of agents can gain by deviating from this proposed pattern of early contracting. This requires the following conditions: (i) it is optimal for agents of types higher than the cutoff type $z$ to choose partners of equal types; (ii) no type $y \geq z$ can gain by contracting with a type $x<z$; (iii) no type $x \geq z$ can gain by contracting with a type $y<z$; and (iv) agents of types lower than $z$ cannot gain by contracting ex ante. Condition i follows directly from the analysis above. Condition ii is satisfied because a type $y$ firm is better off matching with a type $y$ worker at a "price" of $u_{*}(y)$ than with type $x$ at $u_{*}(x)$. Hence a type $y$ firm is better off matching with a type $y$ worker than with type $x$ at $U_{*}(x)>u_{*}(x)$. Condition iii is symmetric to condition ii. For condition iv, it suffices to show that, for any types $x, y<z$, the sum of expected utility to the two agents from contracting ex ante is less than the sum of $U_{*}(x)$ and $V_{*}(y)$. The difference, $\left[U_{*}(x)+V_{*}(y)\right]-w(x, y)$, is given by

$$
\frac{1}{2}(x-y)^{2}+\frac{\left(r_{1}^{2}+r_{2}^{2}\right) \sigma^{2}}{r_{1}+r_{2}}\left(z^{2}-\frac{r_{1}^{2}}{r_{1}^{2}+r_{2}^{2}} x^{2}-\frac{r_{2}^{2}}{r_{1}^{2}+r_{2}^{2}} y^{2}\right),
$$

\footnotetext{
+ When $r_{1}>r_{2}$, a decrease in $r_{2}$ also increases the difference $r_{1}-r_{2}$, but it does not necessarily decrease $z$ because it reduces the demand for insurance by firms as well as increases the opportunity for risk sharing between workers and firms.

"This comparative statics result may change if the uncertainty is mainly on the workers' side. Intuitively, when the uncertainty on the workers' side is too great, even the most promising workers can become unattractive to promising firms because the loss of sorting efficiency is too great. For an analysis of assignment markets with one-sided uncertainty, see Li and Rosen (1998) and Suen (2000). Also, as pointed out in Sec. IV, in this case the pattern of early contracting is likely to be worker-driven, so changes in the pattern of early contracting must also be considered in the relationship between the extent of early contracting and the degree of uncertainty.

${ }^{6}$ Strict inequality holds unless $r_{2}=0$. When firms are risk-neutral, their early contracting payoffs equal the waiting payoffs. In this case, all the gains from risk sharing accrue to workers.
} 
which is positive because $x, y<z$.

\section{Early Contracting with Nonbinding Contracts}

The analysis so far has assumed that early contracts are binding in that agents do not renegotiate after their ability is fully revealed. However, the incentives to renegotiate always exist. Let us consider the extreme case in which all ex ante contracts are nonbinding in that pairs matched by ex ante contracts can exchange partners subject to contractual terms. Since the ex post distributions of $X$ and $Y$ are identical, all assignments will be made between agents with the same ability, the payoff to individuals with ability $X$ will be $X^{2} / 2$, and individuals with ability $Y$ will receive $Y^{2} / 2$. As long as the realized abilities of the pair matched by ex ante contracts differ, which occurs with probability one, the sum of the payoffs the two can get by rematching exceeds what they can produce together, and so it is Pareto-optimal to renegotiate and rematch. ${ }^{7}$ Thus, in the ex ante contract, an optimal linear sharing rule divides the sum of $X^{2} / 2$ and $Y^{2} / 2$. A type $y$ firm's expected utility from ex ante contracting with a type $x$ worker is therefore

$$
v(x, y)=\left(\frac{x^{2}}{2}+\frac{y^{2}}{2}+\sigma^{2}\right)-\frac{r_{1} r_{2}}{r_{1}+r_{2}}\left(x^{2} \sigma^{2}+y^{2} \sigma^{2}+\sigma^{4}\right)-u_{*}(x) .
$$

This expression is separable in $x$ and $y$, which implies that the assignments in the ex ante market are indeterminate. This indeterminacy arises because complementarity in the production function is no longer relevant in the ex ante market. Individuals know that the ex ante match will be broken with probability one after ability is revealed. The only purpose of the ex ante contract is insurance. Since all agents on each side have the same degree of risk aversion, the identity of the ex ante partner is immaterial.

Since $v(x, y)$ is separable in $x$ and $y$, in order for type $y$ to be indifferent among all types of workers, there must exist a set of equilibrium prices $u_{*}(x)$ for workers such that the partial derivative of $v(x, y)$ with respect to $x$ equals zero. Thus

$$
u_{*}(x)=\left(\frac{1}{2}-\frac{r_{1} r_{2} \sigma^{2}}{r_{1}+r_{2}}\right) x^{2}+u_{0}
$$

\footnotetext{
${ }^{7}$ The conclusion that rematching occurs with probability one with nonbinding contracts does not depend on the form of production function. Of course in real-life assignment markets the probability of a rematch is less than one. There may be two reasons. One is that ex post types are discrete rather than continuous as here (see Li and Rosen 1998). The other reason is that rematching is costly.
} 
where $u_{0}$ is an integration constant. The expected utility $v_{*}(y)$ to type $y$ is given by

$$
v_{*}(y)=\left(\frac{1}{2}-\frac{r_{1} r_{2} \sigma^{2}}{r_{1}+r_{2}}\right) y^{2}+\sigma^{2}-\frac{r_{1} r_{2} \sigma^{4}}{r_{1}+r_{2}}-u_{0} .
$$

Since nonbinding contracts allow renegotiation to obtain the ex post efficient assignments, ex ante contracting entails no loss of assignment efficiency. Unlike the case of binding contracts, early contracting will be complete: agents of all types will sign the nonbinding contract to reap the insurance gains. This can be seen by comparing equations (10) and (11) with equations (4) and (5). For example, if

$$
u_{0}=\left(1-\frac{r_{1} r_{2} \sigma^{2}}{r_{1}+r_{2}}\right) \frac{\sigma^{2}}{2}
$$

it can be verified that $u_{*}(x)>U_{*}(x)$ and $v_{*}(y)>V_{*}(y)$ for all $x$ and $y$. Such a $u_{0}$ is of course not unique.

\section{Equilibrium Timing of Early Contracting}

When there are only two possible appointment dates, ex ante and ex post, the uncertainty regarding the ability of agents is resolved between these two dates. However, in some markets, information regarding ability arrives gradually, and there are many possible appointment dates. The timing of market transactions becomes more complicated, and new questions arise. For example, will the date of early contracting unravel back to the very first date on which the market opens? Will all individuals choose to contract at the same date?

To consider such questions, let us consider a simple dynamic structure of uncertainty. At date 0 , all workers are identical and know only that their ability is distributed uniformly on the interval $[0,1]$. At date 1 , some information is revealed so that a worker knows whether his ability lies uniformly in the interval $\left[0, \frac{1}{2}\right]$ or in the interval $\left[\frac{1}{2}, 1\right]$. In general, a type $t$ worker in period $j$ knows that his ability is uniformly distributed on $\left[t-2^{-(j+1)}, t+2^{-(j+1)}\right]$, with $t=(2 k+1) 2^{-(j+1)}\left(k=0,1, \ldots, 2^{j}-\right.$ 1). In period $j+1$, more information is revealed, and this worker will know whether his ability is uniform on $\left[t-2^{-(j+1)}, t\right]$ or uniform on $\left[t, t+2^{-(j+1)}\right]$, with equal probability. The structure of information revelation for firms is the same as that for workers. For simplicity we assume that there is no discounting and that both firms and workers have the 
same risk-aversion parameter $r^{8}$ Consider the contracting equilibrium in which a type $t$ worker contracts with a firm of the same type. Since firms and workers are equally risk-averse, payoff will be equally shared. Thus the expected utility for the worker if he signs an early contract at date $j$ will be

$$
U(t ; j)=\frac{t^{2}}{2}-\frac{r}{4}\left(\frac{t^{2}}{3 \cdot 2^{2 j+1}}+\frac{1}{9 \cdot 2^{4 j+4}}\right) .
$$

If the same individual waits until period $j+1$ to sign the contract, his payoff will be $X_{-} Y_{-} / 2$ with probability one-half and $X_{+} Y_{+} / 2$ with probability one-half, where $X_{-}$and $Y_{-}$are independent uniform random variables on $\left[t-2^{-(t+1)}, t\right]$ and $X_{+}$and $Y_{+}$are independent uniform random variables on $\left[t, t+2^{-(j+1)}\right]$. Expected utility from signing at date $j+1$ is

$$
U(t ; j+1)=\frac{1}{2}\left(t^{2}+\frac{1}{2^{2+3}}\right)+\frac{r}{4}\left(\frac{7 t^{2}}{3 \cdot 2^{2 j+3}}+\frac{7}{9 \cdot 2^{4 j+8}}\right) .
$$

The utility difference between contracting at date $j$ and contracting at date $j+1$ is

$$
U(t ; j)-U(t ; j+1)=-\frac{1}{2^{2 j+5}}+\frac{r}{4}\left(\frac{2^{2 j+5} t^{2}-1}{2^{4 j+8}}\right) .
$$

The first term in this expression is the loss from less efficient sorting, and the second term is the gain from variance reduction as a result of contracting earlier. ${ }^{9}$ A type $t$ individual will sign an early contract at date $j$ instead of waiting for additional new information in the next period if

$$
t^{2}>\frac{1}{r}+\frac{1}{2^{2 j+5}}
$$

Note that the right side of this condition is decreasing in $r$ and in $j$. A type $t$ individual will contract at the earliest date $j$ for which $t^{2}>r^{-1}+$ $2^{-(2 j+5)}$. Agents will contract earlier if they are more risk-averse. Moreover, agents of higher types will sign contracts earlier. As time passes, waiting becomes less attractive relative to contracting, and workers of lower types will decide to stop waiting.

\footnotetext{
${ }^{8}$ The dynamic aspects of early contracting are sensitive to the information structure and to the distribution of ability. Here we use a particularly simple information structure to illustrate how the trade-off between insurance and efficient sorting plays a role in the determination of the timing of early contracting. This model is designed to give explicit solutions; it does not exhaust all the economic forces in a full analysis of a dynamic equilibrium. We leave this as an open question for future research.

${ }^{9}$ Since $t \geq 1 / 2^{j+1}$, the term in parentheses is always positive.
} 


\section{Worker-Driven Early Contracting}

The analysis in previous sections brings us to the following questions. If early contracting results from resolving the trade-off between risk sharing and efficient sorting in favor of risk sharing, does this trade-off always vary with the prospects of workers and firms in the way suggested by the model in Section III, so that early contracting takes the form of top clearing? If the two sides are asymmetric - say workers are risk-averse but firms are risk-neutral so that more promising workers care more about sharing risks while more promising firms care more about sorting efficiency-can promising workers be motivated to sign with not promising firms while less promising workers are forced to wait and more promising firms are content to wait?

In this section, we examine the possibility of a pattern different from top clearing, namely, "worker-driven" early contracting, in which higher types of workers form positive assortative matches with lower types of firms while lower types of workers and higher types of firms wait. We provide conditions conducive for such an early contracting pattern to be a competitive equilibrium: workers are more risk-averse than firms, have greater uncertainty regarding their quality, and face a tighter market, and the production function exhibits increasing returns to the ability of firms. An explicit model that is closely related to the one in Section III further illustrates why and how worker-driven equilibrium can occur. The model also allows us to discuss the distribution of benefits from early contracting. These distribution results are consistent with attempts by firms in some labor markets to ban early contracting, as documented by Roth and Xing (1994). This suggests that worker-driven early contracting is more than just a theoretical possibility in matching markets with uncertainty.

\section{A. Conditions for Worker-Driven Early Contracting Equilibrium}

Suppose that the types of workers and firms are continuously distributed on an interval $[t, \bar{t}]$. Consider an early contracting equilibrium in which an interval $[\underline{x}, \bar{x}] \subset[t, \bar{t}]$ of workers and an interval $[\bar{x}, \bar{y}] \subset[t, \bar{t}]$ of firms of equal size form pairwise matching in the ex ante market. If the equilibrium is worker-driven, then a type $\underline{x}$ worker must be indifferent between signing an ex ante contract with a type $y$ firm and waiting, and types just above $\underline{x}$ prefer signing with type $y$ to waiting. Thus a necessary equilibrium condition is

$$
\frac{\partial w(\underline{x}, \underline{y})}{\partial x} \geq U_{*}^{\prime}(\underline{x})
$$

where the function $w(x, y)$ is given by equation $(1)$, and $U_{*}(x)$ gives the 
expected utility from waiting for any type $x$ worker in the equilibrium. Similarly, a type $\bar{y}$ firm in equilibrium is indifferent between waiting and signing an ex ante contract with a type $\bar{x}$ worker, and types just above $\bar{y}$ prefer waiting to signing with type $\bar{x}$. Thus another necessary equilibrium condition is

$$
\frac{\partial w(\bar{x}, \bar{y})}{\partial y} \leq V_{*}^{\prime}(\bar{y}),
$$

where $V_{*}(y)$ gives the expected utility from waiting for any type $y$ firm in the equilibrium. Note that in a top-clearing contracting equilibrium, the inequality in condition (13) is reversed for some critical type $\chi$, because firms of types higher than $y$ prefer early contracting to waiting, and condition (12) continues to hold for the critical type of worker. Thus a crucial condition for a worker-driven contracting equilibrium, as opposed to a top-clearing equilibrium, is that promising firms must be willing to let less promising types outbid for promising workers.

Using the equilibrium conditions in the ex post market, we can obtain expressions for $U_{*}^{\prime}(x)$ and $V_{*}^{\prime}(y)$. Let $A(Y)$ be the assignment function in the ex post market and $B(X)$ be its inverse, and let $C(X)$ be the equilibrium payoff to a worker with realized ability $X$. Then the first-order condition for firm $Y$ to optimally choose worker $A(Y)$ is

$$
C^{\prime}(A(Y))=F_{1}(A(Y), Y) .
$$

Thus, under the additive specification of conditional distributions, we have

$$
U_{*}^{\prime}(x)=E\left[F_{1}(X, B(X)) \mid x\right]-2 r_{1} \operatorname{Cov}\left[C(X), F_{1}(X, B(X)) \mid x\right]
$$

and

$$
\begin{aligned}
V_{*}^{\prime}(y)= & E\left[F_{2}(A(Y), Y) \mid y\right]-2 r_{2} \operatorname{Cov}[F(A(Y), Y) \\
& \left.-C(A(Y)), F_{2}(A(Y), Y) \mid y\right] .
\end{aligned}
$$

On the other hand, if $a(y)$ is the one-to-one assignment function in the ex ante market and $b(x)$ is its inverse, under the additive specification of conditional distributions, equation (1) implies

$$
\begin{aligned}
\frac{\partial w(x, b(x))}{\partial x}= & E\left[F_{1}(X, Y) \mid x, b(x)\right] \\
& -\left(\frac{2 r_{1} r_{2}}{r_{1}+r_{2}}\right) \operatorname{Cov}\left[F(X, Y), F_{1}(X, Y) \mid x, b(x)\right]
\end{aligned}
$$

and 


$$
\begin{aligned}
\frac{\partial w(a(y), y)}{\partial y}= & E\left[F_{2}(X, Y) \mid a(y), y\right] \\
& -\left(\frac{2 r_{1} r_{2}}{r_{1}+r_{2}}\right) \operatorname{Cov}\left[F(X, Y), F_{2}(X, Y) \mid a(y), y\right]
\end{aligned}
$$

for all types $x$ and $y$ that in equilibrium sign ex ante contracts. Expressions (15)-(18) and the necessary conditions (12) and (13) can help us understand what conditions make worker-driven contracting equilibrium more likely.

The analysis of Section III suggests that for an asymmetric early contracting pattern such as worker-driven contracting to occur, the two sides of the market should be asymmetric. One reasonable asymmetry concerns risk attitudes, in that workers are more risk-averse than firms. Intuition suggests that a worker-driven early contracting equilibrium is more likely to happen if the risk-aversion asymmetry is greater. Riskaverse workers are eager to obtain insurance from ex ante contracts, and higher types of workers can have more at risk from waiting than lower types of workers. Conversely, since firms are not so risk-averse, they are less eager to sign ex ante contracts. Higher types of firms have stronger incentives to wait than lower types to take advantage of more efficient assignments instead of outbidding the lower types for more promising workers. Formally, we note that the covariance term in equation (15) is the derivative of the variance of a type $x$ worker's payoff in the ex post market. If the term is positive, higher types of workers face greater payoff risks from waiting. By equation (14), a sufficient condition for this to be true is that the ex post equilibrium wage $C(X)$ is convex in realized ability $X$. Similarly, if the covariance term in equation (17) is positive, then higher types of workers also face higher payoff risks from signing early contracts. Assume that all the covariance terms in equations (15)-(18) are positive. Then, for workers, a large $r_{1}$ increases the disutility from the payoff risks from waiting and thus reduces $U_{*}^{\prime}(x)$, and a small $r_{2}$ reduces the payoff risks from early contracting and thus increases $u_{*}^{\prime}(x)$. For firms, the opposite occurs: a large $r_{1}$ increases the disutility from the risks in early contracts (if firms are risk-averse) and thus reduces $v_{*}^{\prime}(y)$, and a small $r_{2}$ reduces the disutility from the payoff risks from waiting and thus increases $V_{*}^{\prime}(y)$. These effects make it more likely that the necessary conditions for worker-driven contracting equilibrium (12) and (13) are satisfied.

The necessary conditions (12) and (13) can also help us understand how different degrees of uncertainty regarding ability on the two sides of the market affect the pattern of early contracting. This is an interesting question because the uncertainty is often more important on the workers' side than on the firms' side. We can capture greater uncertainty 
on the workers' side by assuming that the variance $\sigma_{1}^{2}$ of the random variable $\gamma_{1}$ is greater than the variance $\sigma_{2}^{2}$ of $\gamma_{2}$. Consider how the size of $\sigma_{1}^{2}$ and $\sigma_{2}^{2}$ affects (17) and (18), while ignoring their effects on the ex post market. In general, these variance terms affect both the average realized quality of early matches (represented by the first terms in the two expressions) and the variability (the second terms). For the production function $F(X, Y)=X Y$, which exhibits constant returns to ability of both inputs, $\sigma_{1}^{2}$ and $\sigma_{2}^{2}$ affect only the variability in the realized quality of early matches. More precisely, (17) and (18) become

$$
\frac{\partial w(x, b(x))}{\partial x}=b(x)-\frac{2 r_{1} r_{2} x \sigma_{2}^{2}}{r_{1}+r_{2}}
$$

and

$$
\frac{\partial w(a(y), y)}{\partial y}=a(y)-\frac{2 r_{1} r_{2} y \sigma_{1}^{2}}{r_{1}+r_{2}} .
$$

Then, if $\sigma_{2}^{2}$ is much smaller than $\sigma_{1}^{2}, \partial w(x, b(x)) / \partial x$ tends to be great and early contracting becomes more attractive to workers, whereas $\partial w(a(y), y) / \partial y$ tends to be small and early contracting becomes less attractive to firms. ${ }^{10}$ As a result, the necessary conditions for worker-driven contracting equilibrium (12) and (13) are more likely to hold.

The balance of supply on the two sides of the market can also affect the pattern of early contracting. In a market with a greater supply of all types of workers, worker-driven early contracting becomes more likely because an excess supply of workers in the ex post market gives higher types of workers more incentives to contract early and higher types of firms more incentives to wait. For example, if the production function is $F(X, Y)=X Y,(15)$ and (16) become

$$
U_{*}^{\prime}(x)=E[B(X) \mid x]-2 r_{1} \operatorname{Cov}[C(X), B(X) \mid x]
$$

and

$$
V_{*}^{\prime}(y)=E[A(Y) \mid y]-2 r_{2} \operatorname{Cov}[A(Y) Y-C(A(Y)), A(Y) \mid y] .
$$

Then a greater supply of all types of workers tends to increase $A(Y)$ for any $Y$ and decrease $B(X)$ for any $X$, causing a downward shift in the ex post wage function $C(X)$ and a corresponding upward shift in firms' payoff function $A(Y) Y-C(A(Y))$. The increase in the supply of workers will directly affect the average level of payoff from the ex post market (represented by the first terms in the two expressions above), but not necessarily its variability (the second terms). Thus $U_{*}^{\prime}(x)$ decreases and

\footnotetext{
${ }^{10}$ Note that the effects of $\sigma_{1}^{2}$ and $\sigma_{2}^{2}$ disappear if firms are risk-neutral because workers are fully insured against risks from early contracting.
} 
$V_{*}^{\prime}(y)$ increases, and it becomes easier to satisfy the two necessary conditions for worker-driven contracting equilibrium (12) and (13).

The last condition for worker-driven early contracting that we identify concerns the production function. In a standard assignment model without uncertainty, the only property of the production function that matters to the matching pattern is positive cross derivatives. In our model of early contracting, the output elasticities of the ability of workers and firms also matter to the matching pattern of early contracting, because they determine the sorting efficiency gains from waiting relative to signing early contracts. In particular, increasing returns to the ability of firms give more incentives to higher types of firms to wait in order to exploit efficient sorting in the ex post market and therefore make worker-driven early contracting more likely. To focus on the production function, we assume that the two random variables $\gamma_{1}$ and $\gamma_{2}$ have identical distributions and that the ex ante distributions of types of the two inputs are identical. Under these assumptions, the ex post distributions of ability are the same for the two inputs if agents of the same types contract with each other in the ex ante market. Our next result gives a necessary condition on the production function for worker-driven early contracting to be an equilibrium when firms are risk-neutral.

Proposition 2. Suppose that firms are risk-neutral. There does not exist worker-driven contracting equilibrium if

$$
E\left[F_{2}(X, Y) \mid y, y\right] \geq E\left[F_{2}(Y, Y) \mid y\right]
$$

for all $y \in[t, \bar{t}]$.

Proof. Since $r_{2}=0$, equation (16) implies that

$$
V_{*}^{\prime}(y)=E\left[F_{2}(A(Y), Y) \mid y\right] .
$$

Suppose that there is a worker-driven early contracting equilibrium. Since the distribution of ability of a higher type stochastically dominates that of a lower type, the ex post assignment function satisfies $A(Y) \leq$ $Y$ for all realized ability $Y$. Since $F_{12}>0$, equation (19) implies that

$$
V_{*}^{\prime}(y) \leq E\left[F_{2}(Y, Y) \mid y\right] \text {. }
$$

Similarly, when $r_{2}=0$, equation (18) simplifies to

$$
\frac{\partial w(x, y)}{\partial y}=E\left[F_{2}(X, Y) \mid x, y\right] .
$$

In the worker-driven equilibrium, $a(y)>y$ for any type $y$ firm that signs an ex ante contract, and therefore

$$
\frac{\partial w(x, y)}{\partial y}>E\left[F_{2}(X, Y) \mid y, y\right]
$$


for any such firm. Under the assumption that

$$
E\left[F_{2}(X, Y) \mid y, y\right] \geq E\left[F_{2}(Y, Y) \mid y\right]
$$

for all $y \in[t, \bar{t}]$, equations (20) and (21) contradict the necessary condition (13). Q.E.D.

Consider again the multiplicatively separable production function given by equation (3). In this case,

$$
\begin{gathered}
E\left[F_{2}(Y, Y) \mid y\right]-E\left[F_{2}(X, Y) \mid y, y\right] \\
=E\left[\theta(Y) \phi^{\prime}(Y) \mid y\right]-E[\theta(Y) \mid y] E\left[\phi^{\prime}(Y) \mid y\right] .
\end{gathered}
$$

Then, the condition in proposition 2 is equivalent to $\phi^{\prime} \leq 0$, or the production function exhibits nonincreasing returns in $Y$. The intuition of proposition 2 is straightforward. If $E\left[F_{2}(X, Y) \mid y, y\right] \geq E\left[F_{2}(Y, Y) \mid y\right]$, higher types of firms can be said to be "predisposed" toward signing early contracts in that they weakly prefer signing early contracts to remaining in the market if early contracting is top clearing. Worker-driven early contracting changes the ex post distributions to the disadvantage of the firms that remain in the ex post market so that higher types of firms have stronger incentives to sign early contracts. In this case, higher types of firms will outbid lower types to sign more promising workers, and so worker-driven equilibrium cannot occur.

\section{B. A Model of Worker-Driven Early Contracting}

The setup of this model is the same as the one in Section IIIB, except that the production function is given by $F(X, Y)=X Y^{2}$ instead of $X Y$ and firms are assumed to be risk-neutral. Note that the model satisfies two of the four conditions identified in Section IVA, namely, indifference to payoff risks on the firms' side and increasing returns to the ability of firms. The latter feature is crucial for the construction of a worker-driven early contracting equilibrium in this model. Recall that by proposition 2 there is no worker-driven early contracting equilibrium in this model if the production function is $X Y$ as in the model of Section III $B$. In that case, firms are predisposed toward neither signing ex ante contracts nor waiting for the ex post market, and since a worker-driven early contracting equilibrium changes the ex post distributions of ability to the favor of workers who remain in the ex post market, higher types of firms will not let lower types outbid them for more promising workers. On the other hand, when the production function is $X Y^{2}$, there is no top-clearing early contracting equilibrium because increasing returns to 
the ability of firms imply that higher types of firms are predisposed toward waiting because of greater gains from efficient sorting. ${ }^{11}$

We assume that the types for the two inputs are distributed identically and uniformly over an interval $[t-1, t]$. As in the model of Section III $B$, for both sides of the market, the ability conditional on a type is normally distributed, with the mean equal to the type and the variance equal to a constant $\sigma^{2}$. We construct a worker-driven equilibrium with positive assortative matches between workers of types $t-z$ to $t$ and firms of types $t-1$ to $t-1+z$, where $z$ is a number between zero and one that represents the extent of early contracting, to be determined as part of the equilibrium.

Since the type distributions are identically uniform for the two inputs, the ex ante equilibrium matching function is given by $a(y)=y+1-$ $z$, with the inverse $b(x)=x-1+z$. Given that abilities of workers and firms are independently distributed, from the definition (1) we have

$$
w(x, y)=x\left(y^{2}+\sigma^{2}\right) .
$$

As firms are risk-neutral, the optimal risk-sharing contracts in the ex ante market give full insurance to workers. Thus firms that sign early contracts face an equilibrium payoff function $u_{*}(x)$ of workers, representing the "ask prices" of workers, such that the payoff to a type $y$ firm that signs an early contract with any type $x$ worker, given by $w(x, y)-$ $u_{*}(x)$, achieves a maximum at $x=y+1-z$. The first-order condition implies a differential equation,

$$
u_{*}^{\prime}(x)=(x-1+z)^{2}+\sigma^{2},
$$

whose solution is

$$
u_{*}(x)=\frac{(x-1+z)^{3}}{3}+\sigma^{2} x+u_{0}
$$

where $u_{0}$ is a constant of integration to be determined. Then the equilibrium payoff function for any type $y$ firm that signs an early contract is

$$
v_{*}(y)=(y+1-z) y^{2}-\frac{y^{3}}{3}-u_{0}
$$

which implies that

\footnotetext{
"Increasing returns to the ability of firms also exclude the existence of "firm-driven" early contracting equilibrium, where higher types of firms contract with lower types of workers while lower types of firms and higher types of workers wait. The reason is that firms are predisposed toward waiting, and a firm-driven early contracting equilibrium would change the ex post distributions of ability to the favor of firms that choose to wait and therefore give even more incentives for higher types of firms to wait.
} 


$$
v_{*}^{\prime}(y)=2 y(y+1-z) .
$$

In the ex post market, since type distributions are identically uniform, the ability distributions of workers and firms differ only in a locational shift by $z$. It follows that the ex post matching function is given by $A(Y)=Y-z$, with the inverse $B(X)=X+z$. By (14), the equilibrium payoff function of workers in the ex post market satisfies

$$
C^{\prime}(X)=(X+z)^{2} .
$$

Note that the payoff function is convex in the realized ability of workers, implying that more promising workers face greater payoff risks. As in the model of Section III $B$, we set the constant of integration such that $C(0)=0$. Then the worker's payoff function is

$$
C(X)=\frac{(X+z)^{3}}{3}-\frac{z^{3}}{3},
$$

and the firm's payoff function is

$$
D(Y)=\frac{2}{3} Y^{3}-z Y^{2}+\frac{z^{3}}{3} .
$$

With some straightforward calculations, we find that the expected waiting payoff function for workers is

$$
\begin{aligned}
U_{*}(x)= & \frac{(x+z)^{3}}{3}+(x+z) \sigma^{2}-\frac{z^{3}}{3} \\
& -r_{1}\left[(x+z)^{4} \sigma^{2}+2(x+z)^{2} \sigma^{4}+\frac{5 \sigma^{6}}{3}\right],
\end{aligned}
$$

with

$$
U_{*}^{\prime}(x)=(x+z)^{2}+\sigma^{2}-4 r_{1}\left[(x+z)^{3} \sigma^{2}+(x+z) \sigma^{4}\right]
$$

and the expected waiting payoff function for firms is

$$
V_{*}(y)=\frac{2 y^{3}}{3}+2 y \sigma^{2}-z y^{2}-z \sigma^{2}+\frac{z^{3}}{3},
$$

with

$$
V_{*}^{\prime}(y)=2 y(y-z)+2 \sigma^{2} .
$$

The necessary conditions (12) and (13) for a worker-driven early contracting equilibrium require that for workers the signing payoff function $u_{*}(x)$ increase faster than the waiting payoff function $U_{*}(x)$ at the critical level $t-z$, and that for firms the signing payoff function $v_{*}(x)$ increase 
slower than the waiting payoff function $V_{*}(x)$ at the critical level $t$ $1+z$. Sufficient conditions to guarantee the two conditions are

$$
u_{*}^{\prime}(x)>U_{*}^{\prime}(x)
$$

for all $x \in[t-1, t]$ and

$$
v_{*}^{\prime}(y)<V_{*}^{\prime}(y)
$$

for all $y \in[t-1, t]$. With the expressions (22)-(25), conditions (26) and (27) are satisfied if

$$
r_{1}>\frac{1}{(t-1)^{2} \sigma^{2}+(t-1) \sigma^{4}}
$$

and

$$
\sigma^{2}>t
$$

These two conditions can be intuitively interpreted. Comparing equation (22) to equation (24), we find that in order for condition (26) to hold, promising workers must benefit sufficiently from risk sharing to overcome the payoff disadvantage from matching with firms that are not good prospects. This is why a lower bound (28) on the risk-aversion parameter of workers is needed for worker-driven equilibrium. On the other hand, comparing equation (23) to equation (25), we find that in order for condition (27) to hold, higher types of firms must gain sufficiently from efficient ex post sorting to forgo the payoff advantage from signing early contracts with promising workers. The gains from efficient sorting to firms (the term $2 \sigma^{2}$ in eq. [25]) are due to the positive correlation between the marginal productivity of firms' ability and their partners' ability, which is greater if the uncertainty regarding ability is greater. This explains why a lower bound (29) on the degree of uncertainty is needed. This also illustrates the point made in proposition 2 that a necessary condition for worker-driven early contracting equilibrium is increasing returns to the ability of firms, because increasing returns generate greater gains from efficient ex post sorting to firms and encourage them to wait instead of outbidding lower types of firms for the promising workers.

With conditions (26) and (27) satisfied, the necessary and sufficient conditions for a worker-driven early contracting equilibrium, where workers of types higher than $t-z$ and firms of types lower than $t-$ $1+z$ form positive assortative early matches, are that there exist $z$ and $u_{0}$ such that the critical agents of type $t-z$ workers and type $t-1+z$ firms are indifferent between contracting early and waiting:

$$
u_{*}(t-z)=U_{*}(t-z)
$$


and

$$
v_{*}(t-1+z)=V_{*}(t-1+z) .
$$

Adding up these two equations and eliminating $u_{0}$, we have a single equation in the unknown $z$ :

$$
u_{*}(t-z)-U_{*}(t-z)=V_{*}(t-1+z)-v_{*}(t-1+z) .
$$

By equations (22) and (24), the left-hand side of equation (32) is decreasing in $z$, and by equations (23) and (25) the right-hand side is increasing in $z$. Therefore, necessary and sufficient conditions for equation (32) to have a unique solution in $z$ are that the left-hand side of equation (32) is greater than the right-hand side at $z=0$ and that the opposite holds at $z=1$. Using the expressions $u_{*}(x), v_{*}(y), U_{*}(x)$, and $V_{*}(y)$, we can show that these conditions are equivalent to an upper bound and a lower bound on risk-aversion parameter $r_{1}$ :

$$
\frac{6 t \sigma^{2}+3 t-6 \sigma^{2}-2}{3 t^{4} \sigma^{2}+6 t^{2} \sigma^{4}+5 \sigma^{6}}<r_{1}<\frac{6 t \sigma^{2}-3 t+1}{3 t^{4} \sigma^{2}+6 t^{2} \sigma^{4}+5 \sigma^{6}} .
$$

It can be shown that condition (28) implies that the upper bound is greater than the lower bound in condition (33) and that as long as $t$ is great enough (it suffices that $t \geq 4$ ), the upper bound above is greater than the lower bound on $r_{1}$ imposed by condition (28). Thus, to find a set of parameters sufficient to imply the existence of a worker-driven early contracting equilibrium, we can first pick any $t \geq 4$, then any $\sigma^{2}$ that satisfies condition (29), and finally any $r_{1}$ that satisfies conditions (28) and (33). We present a numerical example of the parameters found in this way. Let $t=4, \sigma^{2}=5$, and $r=0.0155$. Then the solutions to equations (30) and (31) are $z=0.63$ and $u_{0}=-20.05$. As $r_{1}$ increases within the bounds defined by equations (28) and (33), the relevant solution $z$ increases. Thus the extent of early contracting depends positively on the risk aversion of workers, a comparative statics result that also holds for the model in Section III $B$.

\section{Distribution of Benefits from Early Contracting}

The model above can be compared to the model in Section IIIB to analyze the distribution of benefits from early contracting between the two sides of the market and among agents of different types on the same side. Suppose that it is possible to impose a ban on early contracting so that all agents have to remain in the market until ability is fully revealed. Among the agents, who will support the ban? The answer depends on the effect of the ban on the ex post distributions of ability. Consider what happens when the ban is enforced in the market de- 
scribed in Section IIIB. The ban has no effect on the distributions of ability in the ex post market because the increased supply of different types of workers as a result of the ban is exactly balanced by the increased supply of the same types of firms. The ban will not change any agent's expected utility from waiting. It then follows from the equilibrium conditions of early contracting that the ban can never benefit any agent and will hurt those who sign early contracts in the equilibrium without the ban by depriving them of the benefits of risk sharing.

Conclusions are different if the ban changes the ex post distributions of ability. Consider a market with risk-neutral firms and a worker-driven early contracting equilibrium, as in the model of the present section. A ban on early contracting in this market will benefit the firms of higher types that choose to remain in the market in the worker-driven equilibrium because the ban changes the ex post distributions of ability of the two inputs to the favor of these firms. More precisely, the assignment function $A(Y)$ in the ex post market shifts upward as a result of the ban because it improves the average quality of workers in the market. Equation (19) then implies that the upward shift in $A(Y)$ results in an upward shift of the firms' payoff function. Thus the firms that choose to wait in the worker-driven equilibrium are better off with the ban on early contracting. ${ }^{12}$

The ban has different effects on the workers and on the firms that sign early contracts. For the numerical example of $t=4, \sigma^{2}=5$, and $r=0.0155$, the welfare effects of the ban on early contracting are illustrated in figure 1 . As argued above, promising firms that wait for the ex post market (types $y$ from 3.63 to 4) will benefit from a ban on early contracting. Since the equilibrium payoffs to firms under the workerdriven early contracting and under the ban are continuous functions of firms' types, the most promising firms among those signing early contracts (types y just below 3.63) will also benefit from a ban on early contracting. Indeed, in our numerical example, all firms will benefit. This result may appear counterintuitive at first glance because firms are risk-neutral and on average the firms signing early contracts in equilibrium are matched with workers more promising than those they will get from the ex post market under a ban. However, payoffs in the workerdriven equilibrium to these firms are determined by the terms of early contracts, which are partially determined by their waiting payoffs in the

\footnotetext{
${ }^{12}$ Early contracting often provokes efforts to prohibit it. Li and Rosen (1998) conjecture that incentives of firms to ban early contracting have to do with the noncompetitive nature of the matching programs in existence before the unraveling of appointment dates. In $\mathrm{Li}$ and Rosen's model of homogeneous firms, a ban on early contracting unambiguously hurts firms ex ante because it prevents them from dealing with an expected shortage of qualified workers by exploiting the insurance demand of more promising workers through early contracting. The present model suggests that one need not abandon the competitive analysis to explain why firms have reasons to support a ban on early contracting.
} 
workers

firms

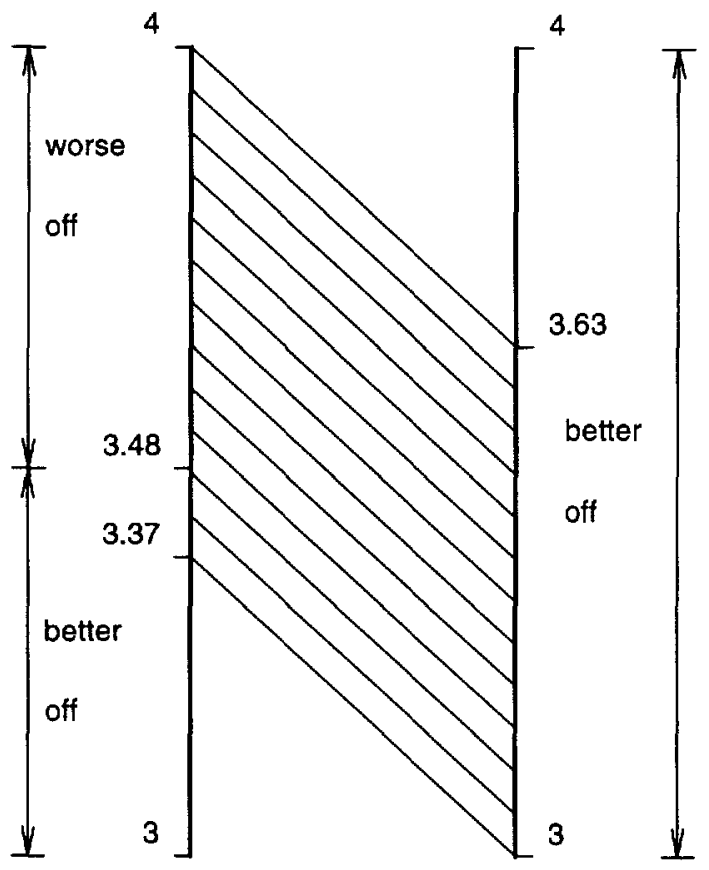

FIG. 1

equilibrium. Since in the work-driven equilibrium any firm faces tougher competition for workers in the ex post market than in the ex post market under a ban, the terms of early contracts are not as favorable to the firms compared to the payoffs from waiting that they will get under a ban.

As for workers, the most promising ones of those signing early contracts (types $x$ from 3.48 to 4 ) will be hurt by a ban on early contracting. Even though under a ban these workers will be matched to more productive firms in the ex post market than their partners in early contracts, the ban deprives them of the benefit of insurance. For exactly the same reason of risk aversion, the less promising workers that wait in the worker-driven equilibrium (types $x$ from 3 to 3.37 ) will benefit from a ban on early contracting. The ban will reduce the average payoff from waiting for these workers compared to the ex post market without a ban, but it will also reduce their payoff risks from waiting. Note that the less promising workers of those contracting early (types $x$ from 3.37 
to 3.48) will also benefit from a ban. As in the top-clearing equilibrium model of Section IIIB, the benefit from risk sharing in early contracting is smaller for these less promising workers, so the ban will benefit them by matching them to more productive firms in the ex post market than their partners in early contracts.

\section{Related Literature}

The marriage model of Gale and Shapley (1962) might seem more appropriate than the assignment model to study college admission mentioned in the Introduction. However, even though there are no explicit money transfers between student applicants and colleges, to the extent that colleges offer different tuition packages, ranking of colleges is not the only determinant in an applicant's preference. The reason we choose the assignment model instead of the marriage model is that we want to apply market analysis to issues related to early contracting. ${ }^{13}$ Our analysis applies with a greater force to assignment markets in which payment transfers are explicitly negotiated. For our analysis to apply directly, there must also be "natural" dates on which appointments can be made, and between the dates there must be new information regarding qualifications of candidates and the desirability of job positions. A cursory look at the markets studied by Roth and Xing (1994) suggests that the markets for American law firms, new marketing professors, and Canadian lawyers satisfy these conditions. In all three markets, wage competition plays a greater role in early appointments than in the markets for medical interns and clerkship. Appointment dates in all these markets can be conveniently characterized as "early" and "regular," and between the two dates significant uncertainty regarding candidates" qualifications is resolved. For the American and Canadian markets of lawyers, early appointments are made through summer associate positions at law firms to second- and first-year students, and regular appointments are made after candidates graduate from law schools. For the market of new marketing professors, early appointments can be made at the summer meetings of the American Marketing Association, whereas regular appointments are made a year after, when candidates graduate from business schools.

Difficulties in controlling recruitment dates in markets for new entrants of highly trained professionals and in other markets have been studied by Roth (1984, 1991), Mongell and Roth (1991), and Roth and Xing (1994). Li and Rosen (1998) point out that these difficulties are due to the absence of complete insurance markets. As in the present

${ }^{13}$ For an introduction to the assignment and marriage models, see Roth and Sotomayor (1990). 
paper, Li and Rosen use a two-period assignment model with individual uncertainty in the first period regarding their productive ability. The uncertainty results in aggregate uncertainty regarding the market value of ability, which plays a crucial role in their analysis. In the present paper, we have assumed continuous distributions of types on the two sides of the market so that the ex post distributions of ability for both sides remain continuous. As a result, the assignments in the ex post market and the resulting payments are deterministic functions, and there is no aggregate uncertainty. In markets as large as the one for law firms, aggregate uncertainty is bound to play a limited role, and the analysis in this paper is more appropriate. Another limitation of $\mathrm{Li}$ and Rosen's analysis comes from their homogeneity assumption regarding firms, which makes it difficult to address issues about patterns of early contracting.

The main contribution of this paper is to provide a competitive framework to study issues about early contracting. The idea of uncertainty regarding ability in early appointments is first used by Roth and Xing (1994) in part of their formal analysis of many markets experiencing timing difficulties. Although the timing of market transactions is the common theme, differences between jumping the gun and unraveling in Roth and Xing (1994) and early contracting in our model must be emphasized. According to Roth and Xing, "in virtually all these markets, the problems originated with the incentives that some market participants have to try to 'jump the gun,' and arrange transactions a little earlier than their competitors" (p. 922). Jumping the gun as defined by Roth and Xing does not directly relate to the risk of making an early appointment to someone whose qualifications are not yet established. ${ }^{14}$ Although one part of their formal analysis uses an example of a marriage market to show that, in the presence of uncertainty regarding qualifications of applications prior to the appointment date of a centralized matching program, early appointments can be made in spite of the stability of the program, the risk-sharing aspect of early contracting is absent in their analysis. Indeed, early appointments occur in that example even though participants are all assumed to be risk-neutral, in contrast with our result in Section IIA that risk-sharing incentives are necessary for early contracting to occur in equilibrium. This contrast arises because in their example the terms of contracts are not compet-

\footnotetext{
${ }^{14}$ Indeed, in their formal analysis, which establishes that the exercise of market power is one source of jumping the gun, not only is the risk unnecessary for jumping the gun to occur, but it must be small enough for the argument to go through. The reason is that to exercise its market power, a firm must be able to identify the receptive worker with sufficient accuracy.
} 
itively determined. ${ }^{15}$ Competitive analysis is the crucial difference between early contracting and jumping the gun. ${ }^{16}$

Roth and Xing's (1994) rigorous research on a wide range of markets experiencing difficulties in coordinating the timing of transactions challenges economists for a theory of the evolution of market institutions. Unfortunately, our framework cannot address the evolution of market institutions directly. Indeed, our basic approach is competitive analysis, which assumes away completely the issue about evolution. However, we believe that in markets with natural appointment dates between which significant uncertainty regarding the quality of matches is resolved, a competitive framework, where agents participate in both the market of early appointments and the market of regular appointments, provides a reasonably good description of market behavior. Moreover, our competitive framework yields insights into issues of early contracting such as the terms and the pattern of early contracting and its welfare effects. Finally, the last part of Section III demonstrates how to extend our framework to situations in which there are no fixed appointment dates agreed on by participants and uncertainty regarding quality of matches is resolved continuously. Future research along this line can determine the timing of market transactions in a more general way.

\section{References}

Becker, Gary S. A Treatise on the Family. Cambridge, Mass.: Harvard Univ. Press, 1981 .

Gale, David, and Shapley, Lloyd. "College Admissions and the Stability of Marriage." American Math. Monthly 69 (January 1962): 9-15.

Koopmans, Tjalling C., and Beckmann, Martin J. "Assignment Problems and the Location of Economic Activities." Econometrica 25 (January 1957): 53-76.

Kremer, Michael. "The O-Ring Theory of Economic Development." Q.J.E. 108 (August 1993): 551-75.

$\mathrm{Li}$, Hao, and Rosen, Sherwin. "Unraveling in Matching Markets." A.E.R. 88 (June 1998): 371-87.

Mongell, Susan, and Roth, Alvin E. "Sorority Rush as a Two-Sided Matching Mechanism." A.E.R. 81 (June 1991): 441-64.

Rosen, Sherwin. "The Economics of Superstars." A.E.R. 71 (December 1981): 845-58.

ㄴ. . "Authority, Control, and the Distribution of Earnings." Bell J. Ecom. 13 (Autumn 1982): 311-23.

Roth, Alvin E. "The Evolution of the Labor Market for Medical Interns and Residents: A Case Study in Game Theory." J.P.E. 92 (December 1984): 991-1016.

\footnotetext{
${ }^{15}$ We thank the referee for drawing our attention to this point.

${ }^{16}$ Jumping the gun also has the connotation of chaos and disequilibrium, whereas our competitive framework invokes order and equilibrium. But this does not imply that our framework cannot be used to capture the chaotic and disorderly aspects of coordinating the timing of transactions (see Suen 2000).
} 
"A Natural Experiment in the Organization of Entry-Level Labor Markets: Regional Markets for New Physicians and Surgeons in the United Kingdom." A.E.R. 81 (June 1991): 415-40.

Roth, Alvin E., and Sotomayor, Marilda A. Oliveira. Two-Sided Matching: A Study in Game-Theoretic Modeling and Analysis. Cambridge: Cambridge Univ. Press, 1990.

Roth, Alvin E., and Xing, Xiaolin. "Jumping the Gun: Imperfections and Institutions Related to the Timing of Market Transactions." A.E.R. 84 (September 1994): 992-1044.

Sattinger, Michael. "Assignment Models of the Distribution of Earnings." $J$. Econ. Literature 31 (June 1993): 831-80.

Shapley, Lloyd, and Shubik, Martin. "The Assignment Game I: The Core." Internat. J. Game Theory 1 (February 1972): 111-30.

Suen, Wing. "A Competitive Theory of Equilibrium and Disequilibrium Unraveling in Two-Sided Matching." Rand J. Econ. 31 (Spring 2000): 101-20.

Tinbergen, Jan. "Some Remarks on the Distribution of Labour Incomes." In International Economic Papers No. 1, edited by Alan T. Peacock et al. London: Macmillan (for Internat. Econ. Assoc.), 1951. 